\title{
Dependence of metal surface properties on the valence-electron density in the stabilized jellium model
}

\author{
M. Brajczewska ${ }^{\mathrm{a}, *}$, C. Henriques ${ }^{\mathrm{b}}$, C. Fiolhais ${ }^{\mathrm{a}}$ \\ ${ }^{a}$ Departamento de Física, Centro de Física Computacional, Universidade de Coimbra, P-3004-516 Coimbra, Portugal \\ ${ }^{\mathrm{b}}$ Departamento de Física, Faculdade de Ciências e Tecnologia, Universidade Nova de Lisboa, P-2825-114 Caparica, Portugal
}

\begin{abstract}
The stabilized jellium model is the simplest model which yields realistic results for the physical properties of simple metals. For the surface properties, its single input is the valence-electron density, which is described by the density parameter $r_{s}$. We remark that the surface energy and the work function as a function of $r_{s}$, within that model, are reasonably approximated by power laws and compare that behaviour with similar descriptions found in the literature and with experiment. We also present a simple relationship between the surface energy and the bulk modulus, which is well fitted by the power $-\frac{7}{2}$ of the density parameter (when the effective valence is taken to be $z^{*}=1$ ). Another simple relationship between the work function and the bulk modulus is shown. (C) 2001 Elsevier Science Ltd. All rights reserved.
\end{abstract}

PACS: 68.10.Cr; 68.35. - p; 73.30. + y

Keywords: Simple metals; Surface energy; Work function

\section{Introduction}

The stabilized jellium model [1] has been successfully applied to understand the physics of surfaces [2], slabs [3] and clusters [4] of simple metals. This model has been recently reviewed in Ref. [5].

The surface properties of stabilized jellium are independent of the valence $z$. They are determined only by the average valence electron density in the bulk $\bar{n}=3 /\left(4 \pi r_{s}^{3}\right)$, with $r_{s}$ the density parameter or Seitz radius.

\footnotetext{
* Corresponding author.

E-mail address: marta@teor.fis.uc.pt (M. Brajczewska).
}

The surface energy $\sigma$ and the work function $W$ can be obtained from Lang-Kohn type [6] calculations for the planar surface of stabilized jellium [2], in the local density approximation for exchange and correlation. Within that approach, the surface energy and the work function depend continuously on $r_{s}$ in a non-analytical way. However, we remark here that both results are well approximated by $r_{s}$-power laws. We compare ours with similar power law descriptions found in the literature (some of them having a theoretical justification), and with experiment.

Sixteen simple or sp-bonded metals are considered: $\mathrm{Be}\left(r_{s}=1.87\right), \mathrm{Al}\left(r_{s}=2.07\right), \mathrm{Ga}\left(r_{s}=2.19\right)$, $\mathrm{Sn}\left(r_{s}=2.22\right), \mathrm{Pb}\left(r_{s}=2.30\right)$, In $\left(r_{s}=2.41\right), \mathrm{Tl}$ $\left(r_{s}=2.48\right), \quad \mathrm{Mg} \quad\left(r_{s}=2.65\right), \quad \mathrm{Li} \quad\left(r_{s}=3.24\right), \quad \mathrm{Ca}$ 
$\left(r_{s}=3.27\right), \quad \mathrm{Sr} \quad\left(r_{s}=3.57\right), \quad \mathrm{Ba} \quad\left(r_{s}=3.71\right), \quad \mathrm{Na}$ $\left(r_{s}=3.93\right), \mathrm{K}\left(r_{s}=4.86\right), \mathrm{Rb}\left(r_{s}=5.20\right)$, and $\mathrm{Cs}$ $\left(r_{s}=5.62\right)$. The $r_{s}$ values are all given in bohr.

The stabilized jellium model with effective valence $z^{*}=1$ predicts also the realistic bulk moduli $B$ for all simple metals, which are well fitted by a $r_{s}^{-7 / 2}$ law [7]. We remark that the adimensional ratio $B r_{s} / \sigma$, within that model, is nearly constant for the range of metallic densities. The same happens with the adimensional ratio $B r_{s}^{3} / W$.

\section{Results}

The stabilized jellium surface energy $\sigma$ (in $\mathrm{erg} / \mathrm{cm}^{2}$ ) as a function of the density parameter $r_{s}$ is represented in Fig. 1. Our calculations for the 16 simple metals are well described by the function $\sigma=6937 \times r_{s}^{-2.7}$ obtained by a least-squares fit (solid line). This result should be compared with similar fits made by other authors. For example, some ab initio calculations [8] have been fitted by $\sigma \sim r_{s}^{-3}$, a result which may be understood on the basis of Miedema's model of metal cohesion [9].

On the other hand, the law $\sigma \sim r_{s}^{-2.5}$ has appeared several times in the literature being presented as a kind of empirical law (see, for example, Ref. [10]). A theoretical justification, based on zeropoint energy of plasmons, has been given in Ref. [11], but received strong criticism [12]. The power $-\frac{5}{2}$ of the density parameter $\left(\sigma=5709 \times r_{s}^{-2.5}\right)$ is displayed in Fig. 1 by the dashed line, showing that it also gives a good account of our results.

We have obtained $\sigma=8134 \times r_{s}^{-2.6}$ (not displayed in the figure) as the best fit to the experimental data taken from Ref. [13].

Recently, in Ref. [14], experimental and theoretical surface energies of simple and transition metals have been systematically examined and the case has been made to describe them by $\sigma \sim r_{m}^{-4}$, where $r_{m}$ is the effective density parameter of Ref. [15]. This $r_{m}$ correlates with $r_{s}$ although not in a simple, analytical way.

Fig. 2 shows separately the four contributions to the stabilized jellium surface energy: exchangecorrelation $\left(\sigma_{x c}\right)$, pseudopotential correction $\left(\sigma_{p \text { seudo }}\right)$, electrostatic $\left(\sigma_{e s}\right)$, and non-interacting kinetic $\left(\sigma_{k i n}\right)$. The best fits which we have obtained (not

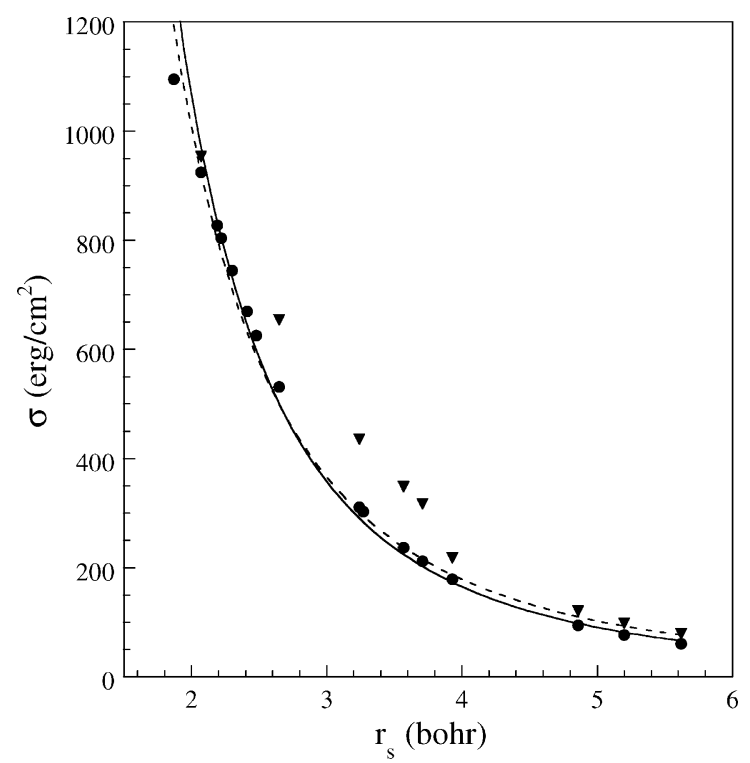

Fig. 1. Surface energy $\sigma$ as a function of the density parameter $r_{s}$. The black circles represent Lang-Kohn results, within the stabilized jellium model, for different simple metals (Be, Al, Ga, $\mathrm{Sn}, \mathrm{Pb}, \mathrm{In}, \mathrm{Tl}, \mathrm{Mg}, \mathrm{Li}, \mathrm{Ca}, \mathrm{Sr}, \mathrm{Ba}, \mathrm{Na}, \mathrm{K}, \mathrm{Rb}$, and $\mathrm{Cs}$ in ascending order of $r_{s}$ ). The solid line represents the power function of $r_{s}$ which better describes those results: $\sigma=6937 \times r_{s}^{-2.7}$. The dashed line shows the best power $-\frac{5}{2}$ fit $\left(\sigma=5709 \times r_{s}^{-2.5}\right)$. The black triangles are experimental surface energies taken from Ref. [13].

displayed in the figure) for those contributions are: $\sigma_{x c}=3.001 \times 10^{4} \times r_{s}^{-3.4}, \sigma_{p s e u d o}=2.584 \times 10^{5} \times r_{s}^{-7.0}$, $\sigma_{e s}=1.160 \times 10^{4} \times r_{s}^{-4.1}$, and $\sigma_{k i n}=-1.554 \times$, $10^{5} \times r_{s}^{-5.1}$. We recall that in the ordinary jellium model, the exchange and kinetic contributions are given by $\sigma_{x} \sim r_{s}^{-3.5}$ and $\sigma_{k i n} \sim r_{s}^{-4.5}$ [16].

Let us now examine the work function. Fig. 3 shows the work function (in $\mathrm{eV}$ ) as predicted by the stabilized jellium model. The best density scaling law $W=6.756 \times r_{s}^{-0.6}$ is indicated by the solid line. As a consequence $\sigma r_{s}^{2} / W \sim r_{s}^{-0.1}$ is almost independent of the density.

The power $-\frac{1}{2}$ of the density parameter was obtained in a recent theoretical study of the work function [17] on the basis of Brodie's definition [18]. We have also tried this power: the dashed line in Fig. 3, representing the best fit $W=6.077 \times$ $r_{s}^{-0.5}$, does not reproduce well our values, namely for the lowest densities. 


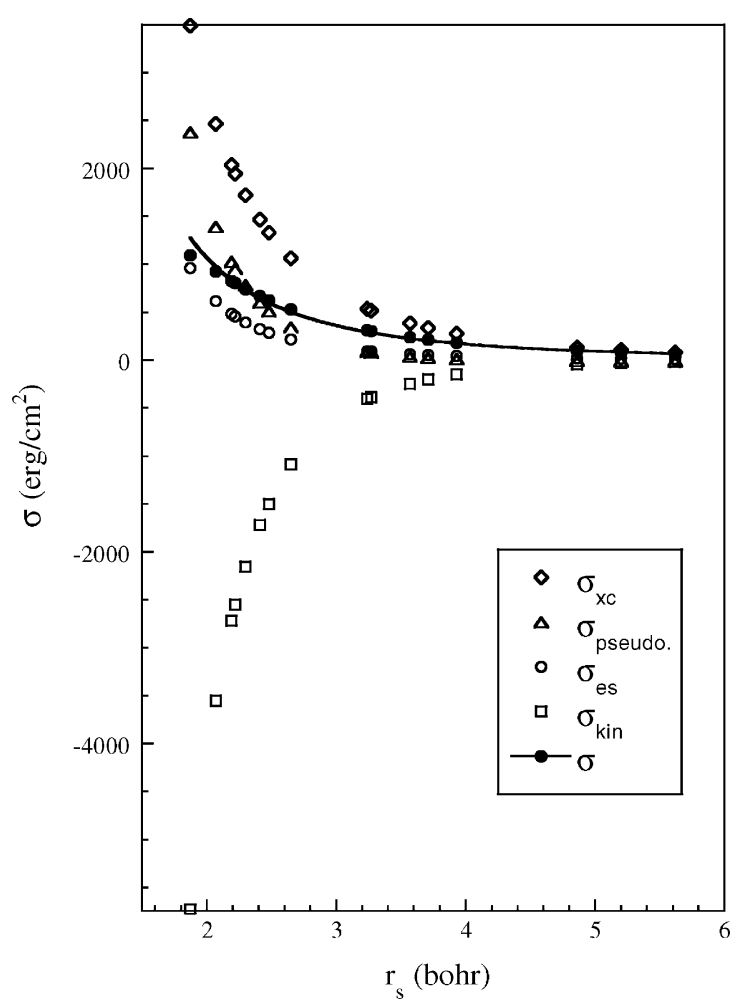

Fig. 2. Surface energy $\sigma$ for different simple metals as predicted by the stabilized jellium model (same as the solid line in Fig. 1) and its decomposition in four contributions: exchange-correlation $\sigma_{x c}$, pseudopotential correction $\sigma_{p s e u d o}$, electrostatic $\sigma_{e s}$, and non-interacting kinetic $\sigma_{k i n}$.

We have obtained $W=7.937 \times r_{s}^{-0.8}$ (not displayed in the figure) as the best fit to the experimental data (polycrystalline samples) taken from Ref. [16].

The stabilized jellium model with effective valence $z^{*}=1$ describes correctly the real dependence of the bulk moduli (or the inverse compressibilities) upon $r_{s}$. Within that model, the bulk moduli are well fitted by $B \sim r_{s}^{-7 / 2}$ [7]. Similar simple power laws can be found in the literature for other kinds of materials. For example, in Refs. [20,21] it is shown that the bulk modulus in covalent materials depends only on the nearest neighbours separation $d$ : $B \sim d^{-7 / 2}$.

It is interesting to relate the bulk and surface problems, analyzing the adimensional ratios $B r_{s} / \sigma$ and $B r_{s}^{3} / W$. From Fig. 4, we observe that $B r_{s} / \sigma$ is nearly constant, approximately equal to 9 . This

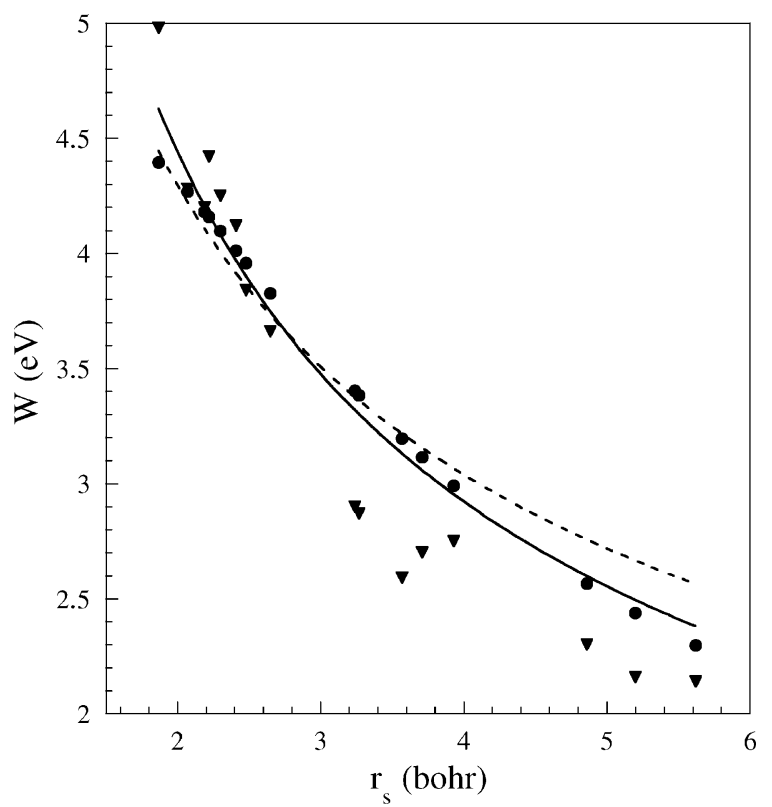

Fig. 3. Work function $W$ as a function of the density parameter $r_{s}$. The black circles represent Lang-Kohn results, within the stabilized jellium model, for different simple metals. The solid line represents the power function of $r_{s}$ which better describes those results: $W=6.756 \times r_{s}^{-0.6}$. The dashed line shows the best power $-\frac{1}{2}$ fit $\left(W=6.077 \times r_{s}^{-0.5}\right)$. The black triangles are experimental work functions taken from Ref. [19].

result agrees with earlier empirical observations that the ratio of the surface energy to the bulk modulus depends only weakly on the material $[22,23]$. A justification for the validity of $B r_{s} / \sigma \simeq$ constant has been given in Ref. [24] using density functional theory in the Thomas-Fermi-DiracWeizsäcker approach. On the other hand, the ratio $\mathrm{Br}_{s}^{3} / W$, also displayed in Fig. 4, is remarkably close to 0.143 for all simple metals.

\section{Conclusions}

In the stabilized jellium model, treated in the framework of Lang-Kohn method using the local density approximation, the surface energy and work function are complicated functions of the density parameter $r_{s}$.

We have shown here that these functions can be well approximated, in the range of metallic densities, by power laws (respectively $\sim r_{s}^{-2.7}$ and 


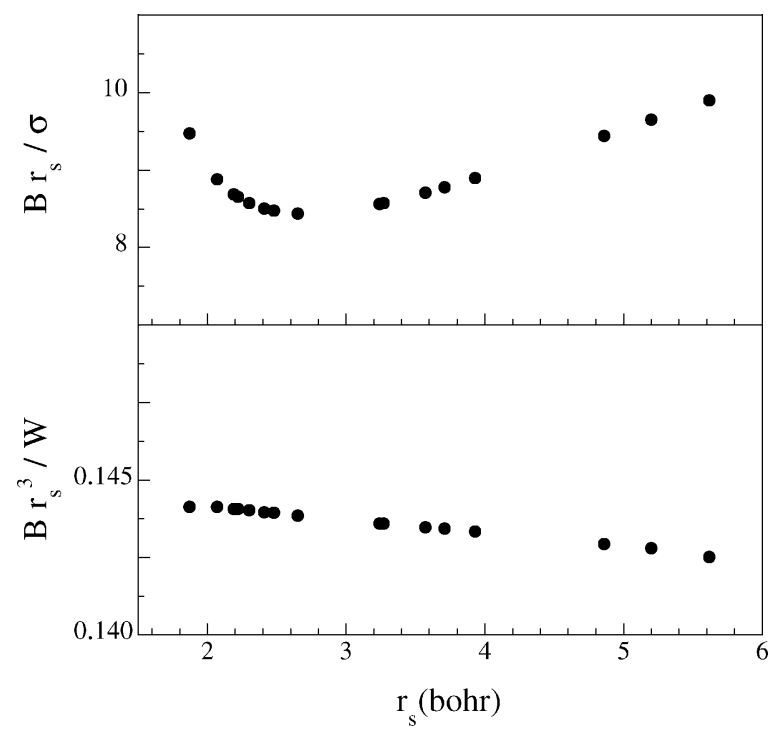

Fig. 4. Adimensional ratios $B r_{s} / \sigma$ and $B r_{s}^{3} / W$ for the simple metals as predicted by the stabilized jellium model, where $B$ is the bulk modulus (calculated with effective valence $z^{*}=1$ ), $r_{s}$ the density parameter, $\sigma$ the surface energy, and $W$ the work function.

$\sim r_{s}^{-0.6}$ ). These relationships, although not exact, are useful to describe the gross trends of surface properties. Of course, they fail outside the range of densities of real metals. For instance, for $r_{s} \leqslant 1.6$ both the surface energy and the work function have maxima.

Since the bulk modulus, in the same model, has also been found to follow a simple power law (with exponent $-\frac{7}{2}$ ), it is possible to combine bulk and surface properties in a way that it is independent of the density. The dimensionless ratios $B r_{s} / \sigma$ and $B r_{s}^{3} / W$ have been found to be approximately constant. Theoretical explanations for these simple results are being investigated.

\section{Acknowledgements}

We thank J. P. Perdew (Tulane University, New Orleans, USA) for having called our attention to the constant value of $B r_{s} / \sigma$ in the stabilized jellium model (with $z^{*}=1$ ). This work has been partially supported by the Portuguese Praxis XXI Program (Project 2/2.1/473/94).

\section{References}

[1] Perdew JP, Tran HQ, Smith ED. Phys Rev B 1990;42:11627.

[2] Fiolhais C, Perdew JP. Phys Rev B 1992;45:6207.

[3] Sarria I, Henriques C, Fiolhais C, Pitarke JM. Phys Rev 2000; 62: 1699.

[4] Seidl M, Perdew JP, Brajczewska M, Fiolhais C. J Chem Phys 1998;108:8182.

[5] Kiejna A. Prog Surf Sci 1999;61:85.

[6] Lang ND, Kohn W. Phys Rev B 1970;1:4555.

[7] Nogueira F, Fiolhais C, Perdew JP. Phys Rev B 1999;59:2570.

[8] Skriver HL, Rosengaard NM. Phys Rev B 1992;46:7157.

[9] Alonso JA, March NH. Electrons in metals and alloys. London: Academic Press, 1989.

[10] Friedel J. Proceedings of the International School of Physics Enrico Fermi. Course LXI, 1976. p. 241.

[11] Schmitt J, Lucas AA. Solid State Commun 1972;11:415.

[12] Vannimenus J. Solid State Commun 1974;15:1739.

[13] Tyson WR, Miller WA. Surf Sci 1977;62:267.

[14] Wojciechowski KF. Surf Sci 1999;437:285.

[15] Perrot F, Rasolt M. J Phys: Condens Matter 1994;6:1473.

[16] Garcia-Moliner F. Introduction to the theory of solid surfaces. Cambridge: Cambridge University Press, 1979.

[17] Halas S, Durakiewicz T. J Phys: Condens Matter 1998;10:10815.

[18] Brodie I. Phys Rev B 1995;51:13660.

[19] Lide DR, editor. CRC Handbook of chemistry and physics. Boston: CRC Press, 1992.

[20] Cohen ML. Phys Rev B 1985;32:7988.

[21] Kelires PC. Phys Rev B 1997;55:8784.

[22] Egelstaff PA, Widom B. J Chem Phys 1970;53:2667.

[23] March NH. Proceedings of the International School of Physics Enrico Fermi. Course LXI, 1976, p. 120.

[24] Brown RC, March NH. J Phys C 1973;6:L363. 